\title{
NcmtRNA Oligonucleotide Andes-1537
}

National Cancer Institute

\section{Source}

National Cancer Institute. ncmtRNA Oligonucleotide Andes-1537. NCI Thesaurus. Code C123825.

A proprietary antisense oligonucleotide targ eting a novel non-coding mitochondrial RNA (ncmtRNA), with potential antineoplastic activity. Upon administration, Andes-1537 binds to ncmtRNA, which is overexpressed in rapidly proliferating cells, such as cancer cells, and not expressed in resting cells. This may decrease the expression of the ncmtRNA, which may inhibit cell proliferation and eventually induce apoptosis in susceptible cancer cells. The proprietary mitochondrial RNA (mtRNA) belongs to the family of non-coding RNAs (ncRNA); it contains an inverted repeat (IR) of 815 nucleotides (nt), which can form a covalent link to the 5' end of the mitochondrial 165 ribosomal RNA (16S mtrRNA). 